\title{
Long-wavelength silicon photonic integrated circuits
}

\author{
G. Roelkens, U. Dave, A. Gassenq, N. Hattasan, C. Hu, B. Kuyken, F. Leo, A. Malik, M. Muneeb, E. \\ Ryckeboer, D. Sanchez, S. Uvin, R. Wang, Z. Hens, R. Baets, Y. Shimura, F. Gencarelli, B. Vincent, R. Loo, J. \\ Van Campenhout, L. Cerutti, J.B. Rodriguez, E. Tournié, X. Chen, M. Nedeljkovic, G. Mashanovich, L. Shen, \\ N. Healy, A. Peacock, X. Liu, R. Osgood, W.M.J. Green
}

\begin{abstract}
In this paper we elaborate on our development of silicon photonic integrated circuits operating at wavelengths beyond the telecommunication wavelength window. Silicon-oninsulator waveguide circuits up to $3.8 \mu \mathrm{m}$ wavelength are demonstrated as well as germanium-on-silicon waveguide circuits operating in the 5-5 $\mu \mathrm{m}$ wavelength range. The heterogeneous integration of III-V semiconductors and IV-VI semiconductors on this platform is described for the integration of lasers and photodetectors operating in the $2-3 \mu \mathrm{m}$ wavelength range. GeSn is proposed as an appealing approach to monolithically integrated long-wavelength detectors. Finally, nonlinear optics in silicon waveguide circuits beyond the two-photon absorption threshold is explored.
\end{abstract}

Index Terms -Silicon photonics, mid-infrared, spectroscopy.

\section{INTRODUCTION}

$\mathrm{S}$ ILICON photonics is an enabling technology for the integration of optical functions, especially in the context of datacommunication and telecommunication. This is mostly related to the mature fabrication processes that are used for the realization of these circuits. In recent years, the range of applications for silicon photonics has extended beyond optical communication and the classical 1.3-1.6 $\mu \mathrm{m}$ wavelength range. Especially in the field of optical spectroscopy there is a need for photonic circuits operating either at shorter wavelengths (visible wavelengths, near-infrared) mostly for fluorescence and Raman spectroscopy. This can be implemented in a silicon photonics context using by using SiN waveguide circuits [1]. Vibrational absorption spectroscopy on the other side requires operation beyond the telecommunication wavelength range, since most molecules have their fundamental rotational-vibrational absorption lines in the mid-infrared.

This work was supported in part by the FP7-ERC-MIRACLE project.

G. Roelkens et al. are with the Photonics Research Group, Ghent University/imec, Belgium \& the center for Nano- and Biophotonics (NBPhotonics), Ghent, Belgium. Y. Shimura et al. are with imec, Belgium. L. Cerutti et al. are with the University of Montpellier 2, France. X. Chen et al. are with the University of Southampton, UK. X. Liu et al. are with the University of Columbia, US. W. Green is with the IBM-TJ Watson Research Center, US.

e-mail: gunther.roelkens@intec.ugent.be

\section{Mid-INFRARED SILICON WAVEGUIDE STRUCTURES}

Silicon-on-insulator waveguide circuits can still be used in the mid-infrared, up to a wavelength of about $4 \mu \mathrm{m}$, after which the absorption of the $\mathrm{SiO}_{2}$ buffer layer becomes the limiting factor [2]. For operation beyond $4 \mu \mathrm{m}$, several platforms have been proposed, including free-standing silicon waveguides, silicon-on-sapphire waveguides or germanium-on-silicon waveguides. In our work we use germanium-of-silicon, given the very wide transparency window of germanium $(2-14 \mu \mathrm{m})$. Both on silicon-on-insulator as on the germanium-on-silicon platform low loss waveguide structures $(3 \mathrm{~dB} / \mathrm{cm}$ at $3.8 \mu \mathrm{m}$ and $5.5 \mu \mathrm{m}$ wavelength respectively) and filter functions (arrayed waveguide gratings, echelle gratings) have been realized, as shown in Figure 1 [3-5].

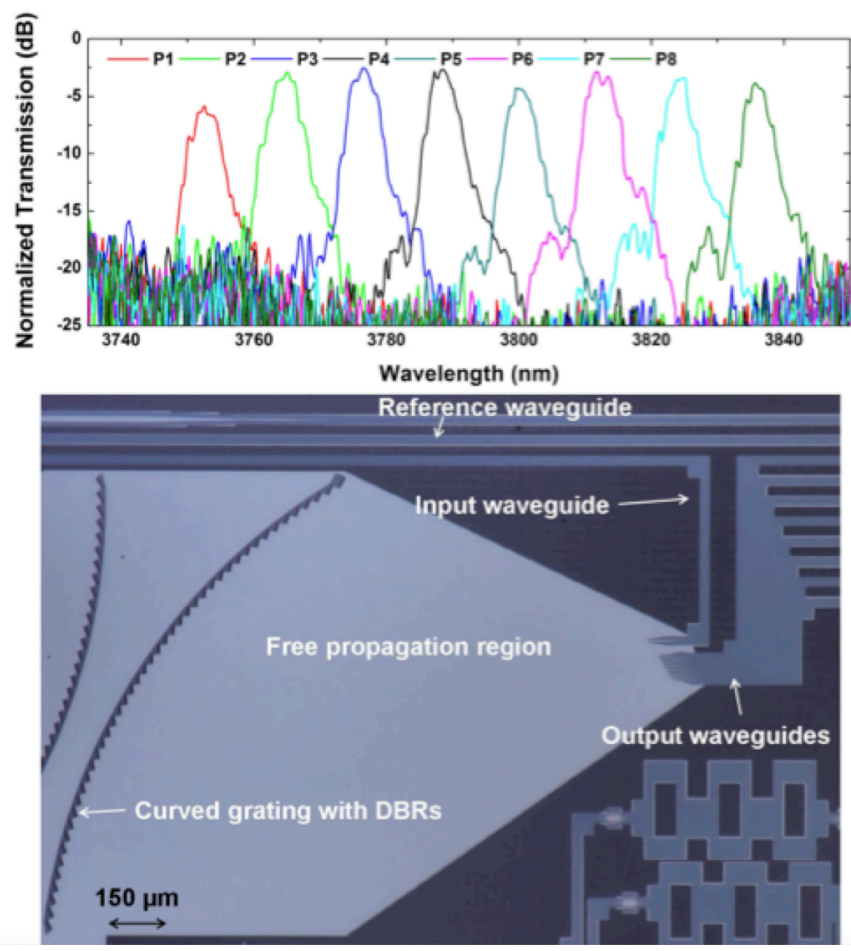

Fig. 1. Normalized transmission spectrum and microscope picture of a planar concave grating operating at $3.8 \mu \mathrm{m}$. 


\section{HETEROGENEOUS INTEGRATION}

In order to go beyond passive silicon waveguide circuits for long-wavelength applications, III-V semiconductor devices and IV-VI semiconductor devices have been heterogeneously integrated on the silicon waveguide platform. These devices include GaSb-based photodetectors with a cut-off wavelength of $2.5 \mu \mathrm{m}$ (responsivity $>1 \mathrm{~A} / \mathrm{W}$ demonstrated) [6] as well as GaSb-based semiconductor lasers operating at $2.4 \mu \mathrm{m}$ [7]. These devices were integrated by means of DVS-BCB bonding, a polymer that is still sufficiently transparent in the 2-3 $\mu \mathrm{m}$ wavelength range. The integration of GaSb-based detector arrays onto silicon-on-insulator spectrometers covering a wavelength range from 1.5 to $2.3 \mu \mathrm{m}$ is shown in Figure 2 [8]. This wavelength range is particularly interesting for the spectroscopic characterization of water-rich fluids.

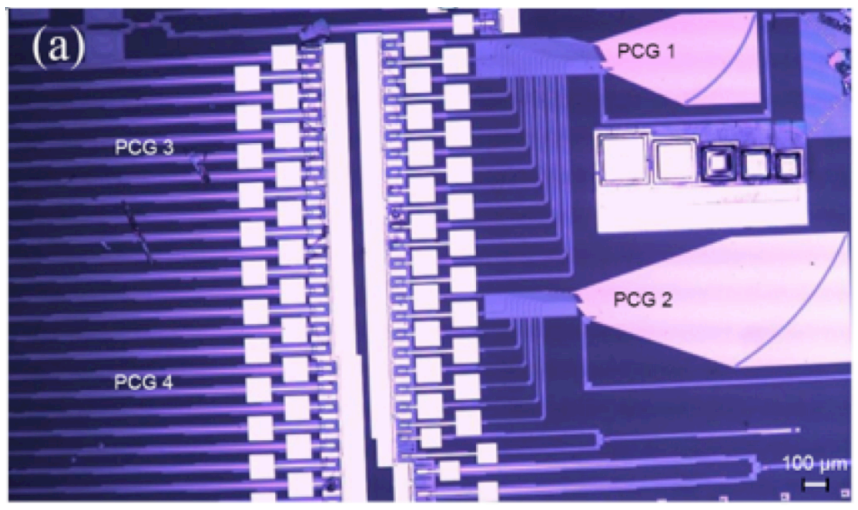

Fig. 1. Microscope picture of the 46 channel integrated spectrometer covering the 1.5-2.3 $\mu \mathrm{m}$ wavelength range;

In order to reduce the cost of integrated long-wavelength detectors, photoconductive devices based on colloidal nanoparticle IV-VI semiconductors ( $\mathrm{PbS}$ and $\mathrm{HgTe}$ ) are being studied, as well as the monolithic integration of GeSn-based photodetectors. The IV-VI semiconductor nanoparticles are synthesized in solution and can be inkjet printed onto the silicon waveguide circuits. The properties of these nanoparticles can be tuned by changing the size of the nanoparticles. PbS nanocrystal photoconductors with a cut-off wavelength of $2.5 \mu \mathrm{m}$ have been demonstrated with a responsivity of several $100 \mathrm{~A} / \mathrm{W}$ due to the large internal gain in these devices. In HgTe photoconductors the wavelength range of operation was extended to about $2.8 \mu \mathrm{m}$ wavelength [9]. Monolithically integrated photodetectors were realized by growing $\mathrm{GeSn} / \mathrm{Ge}$ multiple quantum well structures on a silicon substrate. This way the cut-off wavelength of the detectors can be extended to about $2.4 \mu \mathrm{m}$ (using $20 \mathrm{~nm}$ thick $\mathrm{Ge}_{0.91} \mathrm{Sn}_{0.09}$ quantum wells) [10]. The integration of these detectors with silicon waveguide structures is under way.

\section{NONLINEAR OPTICS}

The tight confinement of the optical field in a silicon-oninsulator waveguide combined with the strong Kerr nonlinearity of silicon make silicon photonic integrated circuits also attractive for nonlinear optics applications. At telecommunication wavelengths the efficiency of the process is however hampered by parasitic two-photon absorption. Moving beyond the two-photon absorption threshold of 2.2 $\mu \mathrm{m}$ greatly reduces the parasitic absorption, allowing the realization of efficient nonlinear processes. Especially the generation of mid-infrared light based on four-wave mixing is of interest. In this context we have demonstrated the generation of $3.6 \mu \mathrm{m}$ radiation based on a telecom signal wavelength and a $2.2 \mu \mathrm{m}$ pump laser, showing that the high refractive index contrast in silicon-on-insulator waveguides does not only help in improving the efficiency of the nonlinear processes, it also allows for dispersion engineering of the silicon waveguide structure in order to reach phase matching over such a wide wavelength span [11]. Supercontinuum generation using short pulse pump sources in the 1.9-2.3 $\mu \mathrm{m}$ wavelength range has also been demonstrated, as will be elaborated on during the conference [12-13].

\section{CONCLUSIONS}

The SOI and germanium-on-silicon platform are ideally suited for the mid-infrared wavelength range, providing ample opportunity for novel and compact devices and systems operating in the mid-infrared. A tantalizing prospect is the realization of complete lab-on-a-chip spectroscopic sensing systems that can be based on various techniques such as absorption spectroscopy and photothermal spectroscopy. Also, the co-integration of 'novel' materials such as graphene and mid-infrared plasmonics can spark the further development of next-generation integrated sensing systems.

\section{REFERENCES}

[1] A. Subramanian et al., "Low-loss singlemode PECVD silicon nitride photonic wire waveguides for $532-900 \mathrm{~nm}$ wavelength window fabricated within a CMOS pilot line," IEEE Photonics Journal, 5(6), p.2202809 (2013) [2] Soref

[3] M. Muneeb et al.,"Demonstration of silicon on insulator mid-infrared spectrometers operating at 3.8um," Optics Express, 21(10), p.1165911669 (2013)

[4] A. Malik et al., "Germanium-on-Silicon Planar Concave Grating Wavelength (de)multiplexers in the Mid-Infrared," Applied Physics Letters, 103(16), p.161119 (2013)

[5] A. Malik et al., "Germanium-on-Silicon Mid-infrared Arrayed Waveguide Grating Multiplexers," Photon. Technol. Lett., 25(18), p.1805-1808 (2013)

[6] A. Gassenq et al., "Study of evanescently-coupled and grating-assisted GaInAsSb photodiodes integrated on a silicon photonic chip," Optics Express, 20(11), p.11665-11672 (2012)

[7] G. Roelkens et al., "Silicon-based photonic integration beyond the telecommunication wavelength range," IEEE Journal of Selected Topics in Quantum Electronics (invited), 20(4), p.8201511 (2014)

[8] E.M.P. Ryckeboer et al. Silicon-on-insulator spectrometers with integrated GaInAsSb photodiodes for wide-band spectroscopy from 1510 to $2300 \mathrm{~nm}$, Optics Express, (2013)

[9] C. Hu, "Short-wave infrared colloidal quantum dot photodetectors on silicon," 2013 Photonics West Conference, 8631, US, paper 8631-79 (2013)

[10] A. Gassenq et al., "GeSn/Ge heterostructure short-wave infrared photodetectors on silicon," Optics Express, 20(25), p.27297-27303 (2012)

[11] B. Kuyken et al., "Generation of 3.6um radiation and telecom-band amplification by four-wave mixing in a silicon waveguide with normal group velocity dispersion," Optics Letters, 39(6), p.1349-1352 (2014)

[12] U.D. Dave et al., "Telecom to mid-infrared spanning supercontinuum generation in hydrogenated amorphous silicon waveguides using a Thulium doped fiber laser pump source," Optics Express, 21(26), p.3203232039 (2013)

[13] B. Kuyken et al.,"Mid-infrared to telecom-band supercontinuum generation in highly nonlinear silicon-oninsulator wire waveguides," Optics Express, 19(21), p.20172-20181 (2011) 